\title{
Artículos de Revisión
}

\section{Resultados maternos y perinatales de los embarazos obtenidos por técnicas de reproducción asistida. Revisión de la literatura.}

\section{Perinatal and maternal outcomes following assisted reproductive technology.}

\section{A literature review.}

\author{
José Moreno Sepúlveda ${ }^{1,2}$, Lucio Ratto ${ }^{3,4}$.
}

${ }^{1}$ Clínica de la Mujer Medicina Reproductiva, Viña del Mar, Chile

2 Departamento de Ginecologia, Universitat Autònoma de Barcelona, España

${ }^{3}$ Departamento de Tocoginecología, Universidad de Buenos Aires, Argentina

${ }^{4}$ Hospital de Clinicas José de San Martin, Buenos Aires, Argentina

Correspondencia: José Moreno - Sepulveda, jmorenos@gmail.com

\section{PRECISE}

Las técnicas de reproducción asistida de alta complejidad se asocian a resultados maternos y perinatales adversos en comparación con embarazos espontáneos

\section{RESUMEN}

La utilización de técnicas de reproducción asistida (TRA) ha aumentado en todo el mundo, incluyendo procedimientos como la donación de gametos, la subrogación y el diagnóstico genético preimplantacional. Creciente evidencia confirma que los embarazos de pacientes sometidas a estos tratamientos tienen un mayor riesgo de complicaciones perinatales. No queda claro si la causa de estos hallazgos se debe a la infertilidad subyacente o los tratamientos en sí. Esta revisión tiene como objetivo resumir la evidencia actual acerca de los efectos sobre los resultados maternos y perinatales tanto de los diferentes procedimientos propios de la fecundación in vitro como de algunos tipos de TRA especiales.

Palabras claves:Técnicas de reproducción asistida, resultados perinatales,bajo peso al nacer, parto prematuro.

\section{ABSTRACT}

The use of assisted reproduction techniques (ART) has increased worldwide, including procedures such as gamete donation, subrogation and preimplantation genetic diagnosis. Growing evidence confirms that pregnancies following these treatments have an increased risk of adverse perinatal outcomes. It is not clear whether the cause of these findings is due to the underlying infertility or the treatments themselves. This review aims to summarize the current evidence regarding the effects of both the different ART procedures and some special types of ART on maternal and perinatal outcomes.

Keywords: Assisted reproductive technology, low birth weight, perinatal outcomes, preterm birth. 


\section{INTRODUCCIÓN}

A lo largo de los años, el principal objetivo de las técnicas de reproducción asistida (TRA) ha sido desarrollar estrategias para maximizar su eficacia, medida a través de la tasa de nacidos vivos ${ }^{1}$. Sin embargo, a medida que se alcanzan resultados cada vez mejores con respecto a este indicador, surge la necesidad de enfocar los esfuerzos en otros aspectos como la seguridad y los costos. Los parámetros propuestos para evaluar la seguridad de una intervención son sus complicaciones, y en el caso de la fecundación in vitro (FIV), una de las más estudiadas es el síndrome de hiperestimulación ovárica (SHO). Aunque el SHO es una complicación potencialmente grave, el desarrollo de nuevas estrategias para mitigar este riesgo ha tenido éxito, como gatillar la ovulación con agonistas de la hormona liberadora de gonadotropinas $(\mathrm{GnRH})$ en vez de gonadotropina coriónica humana ${ }^{2}$.

Otras complicaciones asociadas a la FIV son los resultados maternos y perinatales adversos; y sólo durante la última década se han comenzado a dilucidar los efectos de las TRA sobre ellos, gracias a la publicación de estudios enfocados en esta variable de seguridad ${ }^{3}$.

Los primeros reportes demostraron un mayor riesgo de resultados perinatales adversos en pacientes sometidas a FIV, en comparación con embarazos espontáneos ${ }^{4}$. Una de las posibles explicaciones fue la alta tasa de embarazos múltiples observada en gestaciones obtenidas por FIV, condición relacionada a gran morbilidad perinatal, incluyendo un riesgo seis veces mayor de prematurez 5. En el año 2013 un 41,1\% de los embarazos obtenidos por TRA en Estados Unidos correspondieron a embarazos múltiples, en comparación con sólo el 3,5\% de los nacidos en la población general ${ }^{6}$. Sin embargo, posteriormente se evidenció que el riesgo de resultados perinatales adversos tras la FIV no se explicaría sólo por la incidencia elevada de embarazos gemelares. Diversos estudios revelaron que los embarazos únicos obtenidos por FIV también presentaban una elevada morbilidad obstétrica, incluyendo el parto prematuro (PP) y bajo peso al nacer (BPN) ${ }^{7}$. Las características de los pacientes, incluida la causa de infertilidad ${ }^{8}$, factores de riesgo preconcepcionales ${ }^{9} \mathrm{y}$ efectos propios de la FIV sobre la placenta y el embrión ${ }^{10}$, podrían asociarse con éstos resultados.
Algunos procedimientos propios de la FIV podrían influir directamente sobre estos resultados ${ }^{10}$, como por ejemplo la estimulación ovárica controlada (EOC), la inyección intracitoplasmática de esperma (ICSI), el cultivo embrionario, la vitrificación embrionaria, la transferencia diferida y la biopsia embrionaria para diagnóstico genético preimplantacional (DGP).

Además, recientes estudios han puesto su atención sobre diferentes tipos de TRA de alta complejidad que han aparecido durante las últimas décadas, incluyendo la donación de ovocitos, espermios y embriones; y la gestación subrogada. Estas técnicas podrían asociarse a peores resultados maternos y perinatales ${ }^{7}$.

El objetivo de la presente revisión es proporcionar una síntesis de la evidencia disponible acerca de los efectos sobre los resultados maternos y perinatales tanto de los diferentes procedimientos propios de la FIV como de algunos tipos de TRA de alta complejidad.

\section{PROCEDIMIENTOS PROPIOS DE LA FIV Estimulación ovárica controlada}

La EOC es utilizada habitualmente en la FIV para aumentar la eficacia, justificándose su uso rutinario sobre el hecho de que se necesitan 3.5 ciclos de FIV no estimulados para lograr la misma tasa de nacimientos vivos que un ciclo estimulado ${ }^{11}$.

Distintos estudios se han enfocado en los posibles efectos deletéreos de la EOC sobre los resultados perinatales. Un metaanálisis reciente reportó que la EOC se asoció con un mayor riesgo de PP, con un riesgo relativo $(\mathrm{RR})$ de 1.27 , intervalo de confianza (IC) del 95\% de 1.03-1.58; y BPN (RR 1.95, IC 95\% 1.03-3.67) en comparación con la FIV con ciclo natural ${ }^{12}$. Se postula que los niveles supra fisiológicos de estradiol, producidos por las cohortes ovocitarias que se desarrollan a partir de la EOC, afectarían los procesos de decidualización y placentación en ciclos de FIV en fresco ${ }^{13}$; lo cual determinaría resultados obstétricos adversos, como el PP BPN y preeclampsia 14,15. Un estudio de cohortes evaluó la asociación entre el número de ovocitos obtenidos y riesgos perinatales como el PP y BPN, encontrando un aumento del riesgo en el grupo de pacientes con más de 20 de ovocitos recuperados en comparación con la respuesta normal, definida como 10 a 15 ovocitos recuperados ${ }^{16}$. Estudios posteriores han demostrado que los niveles suprafisiológicos de estradiol 
alcanzados a partir de la EOC aumentan también el riesgo de PP y BPN en embarazos obtenidos a partir de ciclos con transferencia diferida; concluyendo que su efecto va más allá del endometrio, pudiendo afectar directamente a la cohorte ovocitaria ${ }^{17,18}$.

\section{Inyección intracitoplasmática de esperma (icsi)}

Uno de los primeros estudios de cohorte retrospectivo que comparó los resultados perinatales en nacimientos únicos obtenidos por ICSI no encontró diferencias significativas en cuanto a PP (odds ratio (OR) 1.17, IC 95\% 0.95-1.46) y BPN (OR 1.14, IC 95\% 0.91-1.44) comparado con concepciones naturales ${ }^{19}$, lo cual fue confirmado posteriormente con una gran cohorte de niños nacidos por ICSI. Si bien no se observaron diferencias en embarazos únicos obtenidos por ICSI versus embarazos espontáneos en cuanto a BPN; si se reportó un menor riesgo al comparar embarazos espontáneos versus ICSI con espermios obtenidos por recuperación espermática testicular (OR 0.58, IC del 95\% 0,44$0,77)^{20}$. Un reciente estudio de cohorte retrospectivo de 141030 pacientes estadounidenses reportó tasas más elevadas de defectos congénitos en embarazos obtenidos por ICSI en comparación con la FIV convencional $(3.0 \%$ versus $2.5 \%$ respectivamente; OR 1.2, IC 95\% 1.2-1.3) ${ }^{21}$. Tomando en cuenta esos resultados, y que aproximadamente la mitad de las ICSI se realizaron en parejas infértiles sin factor masculino; los autores advierten que la ICSI podría estar siendo indicada en exceso ${ }^{21}$.

\section{Cultivo extendido y transferencia de blastocistos}

Durante la última década se ha producido en todo el mundo un aumento exponencial de los ciclos con transferencia embrionaria en etapa de blastocisto, en desmedro de la transferencia de embriones en etapa de clivaje ${ }^{22}$. Las primeras revisiones sistemáticas y metanálisis reportaron que la transferencia de blastocistos se asoció con un mayor riesgo de PP comparado con la transferencia en etapa de clivaje; sin embargo, destacaron que este efecto sólo se observa al comparar transferencias embrionarias en fresco; y que otras variables confusoras, como el número de embriones transferidos, podrían afectar los resultados de forma importante ${ }^{23,24}$. La transferencia de blastocistos se ha asociado además con un riesgo mayor de nacidos grandes para la edad gestacional, a la vez que la transferencia en etapa de clivaje con un riesgo mayor de nacidos pequeños para la edad gestacional $^{23}$.

Recientes publicaciones basadas en grandes cohortes retrospectivas han realizado seguimientos intentando aislar el factor criopreservación, reportando que luego de transferir blastocistos en fresco, no existen diferencias en cuanto al riesgo de PP (RR 1.00, IC 99.5\% 0.79-1.25), BPN (RR 0.92, IC $99.5 \% 0.73-1.16)$ y malformaciones congénitas (RR 1.16, IC 99.5\% 0.90-1.49) comparado con la transferencia en etapa de clivaje ${ }^{25}$; lo que contrasta con otro estudio que sí observó un mayor riesgo de PP (OR 1.33, IC 95\% 1.09-1.62) luego de transferir blastocistos vitrificados; concluyendo que factores asociados a la criopreservación tendrían un mayor efecto sobre los resultados perinatales que el cultivo extendido a blastocisto ${ }^{26}$. Distintos cambios epigenéticos inducidos por la criopreservación podrían afectar al embrión y con ello los resultados perinatales ${ }^{27}$.

\section{Vitrificación embrionaria}

Los primeros protocolos de criopreservación utilizaron la congelación lenta de embriones en etapa de clivaje. Posteriormente, la introducción de medios de cultivo más complejos permitió la generación de embriones de mejor calidad, extendiendo el cultivo hasta la etapa de blastocisto, lo cual junto al desarrollo de técnicas de vitrificación, han determinado que actualmente la técnica de criopreservación más utilizada sea la vitrificación de blastocistos ${ }^{28}$.

Inicialmente existió preocupación con respecto a la seguridad de la vitrificación, debido a las altas concentraciones de crioprotectores potencialmente tóxicas requeridas y la exposición de los embriones a contaminantes ambientales ${ }^{29}$; sin embargo surgieron nuevos métodos de vitrificación más seguros. Estudios retrospectivos previos han reportado que los embarazos a partir de FIV con congelación lenta versus vitrificación no presentan diferencias en cuanto a la incidencia de PP, mortalidad perinatal, SHE, diabetes, placenta previa y desprendimiento placentario ${ }^{30}$, ni tampoco un efecto sobre el peso al nacer ${ }^{31}$. Estos hallazgos fueron confirmados recientemente por un estudio de cohorte retrospectivo multicéntrico de 3199 nacidos únicos, reportando que la vitrificación no se asoció con un mayor riesgo de resultados adversos en comparación con la congelación lenta, incluyendo PP, BPN y macrosomía 32. En este estudio se controlaron variables 
confusoras importantes, como el sexo masculino, puesto que el posible efecto de la criopreservación sobre el peso al nacer podría ser potencialmente específico en el sexo masculino ${ }^{33}$. Considerando su superioridad en cuanto a las tasas de nacidos vivos y la no inferioridad en los resultados perinatales, la transición desde la congelación lenta a la vitrificación de embriones resulta tranquilizadora.

\section{Transferencia diferida}

Una reciente revisión sistemática y metanálisis reportó que embarazos únicos obtenidos por transferencia diferida presentaron un menor riesgo de PP (RR 0.90; IC 95\% 0.84-0.97) y BPN (RR 0.72; IC 95\% 0.67-0.77) comparado con la transferencia de embriones en fresco; sin embargo presentaron un mayor riesgo de síndrome hipertensivo del embarazo (SHE) (RR 1.29; IC 95\% 1.07-1.56) y nacidos grandes para la edad gestacional (RR 1.54; IC 95\% 1.48-1.61) ${ }^{34}$. Un metanálisis de ensayos clínicos randomizados confirmó un riesgo mayor de preeclampsia (RR 1.79; IC 95\% 1.03-3.09) luego de la transferencia diferida; no obstante, no reportó diferencias en cuanto al PP ${ }^{35}$.

Nuevos ensayos clínicos randomizados han buscado identificar estos riesgos en diferentes poblaciones de pacientes sometidas a FIV con transferencia diferida versus transferencia en fresco, reportándose un mayor riesgo de preeclampsia y nacidos grandes para la edad gestacional con transferencia diferida tanto en pacientes normo ovulatorias 36 como en anovulatorias ${ }^{37}$, grupo asociado a una hiperrespuesta a la EOC.

Los malos resultados observados tras la transferencia en fresco podrían explicarse por los efectos de la EOC explicados más arriba; mientras que los asociados a la transferencia diferida se atribuyen a factores inducidos por la criopreservación y la preparación endometrial artificial sobre la placentación, probablemente por mecanismos epigenéticos y hormonales ${ }^{38}$.

\section{Biopsia embrionaria y diagnóstico genético preimplantacional}

El uso del DGP ha aumentado sostenidamente, principalmente debido a la reducción en el riesgo de abortos y embarazos con aneuploidías, reducción del tiempo necesario para conseguir un nacido vivo, y permitir que parejas portadoras de patologías asociadas a la mutación de un solo gen tengan un hijo no afectado ${ }^{39}$. A pesar de estas ventajas, recientes estudios se han enfocado en determinar los riesgos potenciales de realizar una biopsia embrionaria de trofoectodermo en etapa de clivaje o blastocisto sobre los resultados perinatales. Un estudio de cohorte retrospectiva no encontró diferencias en cuanto al riesgo de PP (OR 0.66, IC 95\% 0.45-0.98) o BPN (OR 0.58, IC 95\%: 0.38-0.88) en embarazos obtenidos por FIV con DGP comparado con FIV sin DGP ${ }^{40}$; lo cual fue confirmado en un estudio posterior ${ }^{39}$, el cual sin embargo reportó un riesgo elevado de preeclampsia (OR 3.02, IC 95\% 1.10-8.29) en el grupo de FIV con biopsia de trofoectodermo. La remoción de células de trofoectodermo, que están destinadas a formar la placenta, podría estar relacionada con un desarrollo placentario anormal y la aparición de preeclampsia, elemento que debe ser explorado en futuros estudios.

\section{TRA ESPECIALES DE ALTA COMPLEJIDAD Donación de ovocitos}

La donación de ovocitos (DO) es una alternativa de tratamiento en mujeres de edad avanzada o con baja reserva ovárica, reportandose elevadas tasas de éxito ${ }^{41}$. En los últimos años, se ha observado un aumento en el número de ciclos de DO realizados en todo el mundo, alcanzando un 15,4\% del total de ciclos en Latinoamérica en $2016^{42}$ y un $7,2 \%$ de los ciclos en Europa en 2014, destacando España con un $27,9 \% 43$.

Los embarazos obtenidos por DO se han asociado con un mayor riesgo de resultados maternos y perinatales adversos en comparación con embarazos espontáneos ${ }^{44}$. Una reciente revisión sistemática y metanálisis reportó que los embarazos obtenidos por DO presentan un riesgo mayor de SHE (OR 2.63, IC $95 \% 2.17-3.18$ ), preeclampsia (OR 2.64, IC 95\% 2.29-3.04), preeclampsia severa (OR 3.22, IC 95\% 2.30-4.49), PP (OR 1.57, IC 95\% 1.33-1.86) y BPN (OR 1.25, IC 95\% 1.20-1.30) comparado con los embarazos obtenidos por FIV autóloga ${ }^{45}$. El mayor riesgo de trastornos hipertensivos podría estar asociado a factores como la edad materna avanzada y una mayor prevalencia de patologías médicas, a la vez que a defectos en la placentación inducidos por la preparación endometrial artificial y una eventual incompatibilidad inmunológica a antígenos derivados del feto ${ }^{46}$. 


\section{Donación de espermios}

La evidencia en relación a este aspecto es antigua y escasa, observándose un mayor riesgo de SHE con semen donado ${ }^{47}$. Se postula la hipótesis de que exponerse al semen paterno antes de la concepción tendría un efecto protector, mientras que el uso de semen donado posterior a un embarazo con semen paterno podría aumentar el riesgo de preeclampsia a través de un mecanismo inmune similar a la donación de ovocitos.

Un reciente metanálisis de estudios observacionales mostró que los nacidos a través de semen donado no presentaron mayor riesgo de PP, BPN o malformaciones congénitas versus nacidos de embarazos espontáneos ${ }^{48}$.

\section{Donación de embriones}

El alza de los ciclos de FIV ha generado un mayor número de embriones criopreservados residuales, aumentando los ciclos con donación embrionaria. No obstante, se conoce poco sobre los resultados de estos embarazos. Un estudio retrospectivo estadounidense de 6773 ciclos de embriodonación, reportó que del total de nacidos vivos, 1,482 (76.8\%) fueron de término y peso adecuado, acorde con lo reportado en FIV convencional ${ }^{49}$. Es difícil concluir el riesgo obstétrico de la embriodonación pues existen muchas variables confusoras como las comorbilidades de la receptora, intentos de FIV fallidos, tiempo de infertilidad y el que en su mayoría los embriones donados son congelados.

\section{Gestación subrogada}

La gestación subrogada consiste en realizar una FIV utilizando los gametos de los padres, donantes o ambos, y que luego se transfieren a otra receptora gestacional ${ }^{50}$. En los últimos años se ha observado un aumento en los ciclos de subrogación en ciertas regiones del mundo debido la legalización de la subrogación en ciertos países y al turismo de subrogación asociado. Un estudio realizado en algunos estados de EEUU, donde la subrogación comercial está permitida, reportó que los ciclos de subrogación se cuadruplicaron entre 1999 a 2013, incluyéndose entre las causas más frecuentes: ciclos previos de FIV fallidos, parejas masculinas del mismo sexo, edad materna avanzada y patologías médicas que hacen que el embarazo sea inseguro ${ }^{51}$.

Sin embargo, la evidencia disponible en relación a los resultados perinatales es limitada y contradictoria.
Una revisión sistemática de 2016 mostró tasas similares de SHE, PP y defectos congénitos al comparar gestaciones subrogadas con FIV autóloga 52, en concordancia con estudio posterior, que utilizando una gran base de datos del Reino Unido tampoco reportó diferencias significativas en cuanto al riesgo de PP 0 anomalías congénitas 53 . Contrariamente, un estudio anterior reportó que las pacientes sometidas a gestación subrogada presentaron un riesgo menor de metrorragia del tercer trimestre, hipertensión inducida por el embarazo y tener un recién nacido BPN ${ }^{54}$.

Sin embargo, al comparar los embarazos obtenidos por gestación subrogada con sus propias concepciones espontáneas previas, un estudio demostró un mayor riesgo de resultados adversos, incluyendo una mayor tasa de gemelos, PP, BPN, diabetes gestacional, SHE y hemorragia posparto 55 . Adicionalmente, un reciente estudio holandés reportó que la incidencia de SHE en las gestaciones subrogadas fue del $20,6 \%$, el doble de lo esperado ${ }^{56}$.

El impacto de la FIV en estos riesgos es incierto, sin embargo es importante destacar que los ciclos de gestación subrogada se asocian con otros factores que pueden influir sobre los resultados perinatales adversos, incluyendo una mayor proporción transferencia de embriones congelados, ovocitos de donantes, biopsia de trofoectodermo para diagnóstico genético preimplantacional y transferencia de 2 embriones en comparación con ciclos autólogos de FIV. ${ }^{57}$. Futuros estudios deben contar con un mayor número de pacientes y ajuste de variables confusoras.

\section{CONCLUSIONES}

Las pacientes con embarazos obtenidos por FIV, autóloga o con donación de gametos, deben estar claramente informadas del mayor riesgo de complicaciones asociadas, y que éste aumento del riesgo materno y perinatal es independiente del embarazo múltiple.

Se requiere de un cuidadoso control preconcepcional a fin de poder optimizar el estado de salud de los pacientes que se someten a FIV, identificando y tratando aquellos trastornos modificables. Además, se debe hacer un esfuerzo para optimizar la seguridad de la FIV en sus distintas fases; controlando aspectos cruciales como la EOC, prevención del $\mathrm{SHO}$ o transferencia de un embrión único, a fin de prevenir y reducir las complicaciones. Finalmente, se requieren estudios prospectivos con 
grandes cohortes y ensayos clínicos randomizados que permitan aclarar la contribución de cada factor sobre los resultados maternos y perinatales.

\section{REFERENCIAS}

1. Barnhart KT. Live birth is the correct outcome for clinical trials evaluating therapy for the infertile couple. Fertil Steril. 2014;101:1205-8.

2. Devroey P, Polyzos NP, Blockeel C. An OHSS-free clinic by segmentation of IVF treatment. Hum Reprod 2011;26:2593-7.

3. Barnhart KT. Assisted reproductive technologies and perinatal morbidity: interrogating the association. Fertil Steril. 2013;99:299-302.

4. Helmerhorst FM, Perquin DA, Donker D, Keirse MJ. Perinatal outcome of singletons and twins after assisted conception: a systematic review of controlled studies. BMJ. 2004 Jan $31 ; 328(7434): 261$.

5. Chambers GM, Ledger W. The economic implications of multiple pregnancy following ART. Semin Fetal Neonatal Med 2014;19:254-61.

6. Sunderam S, Kissin DM, Crawford SB, Folger SG, Jamieson DJ, Warner L, Centers for Disease Control and Prevention (CDC), et al. Assisted Reproductive Technology Surveillance-United States, 2013. MMWR Surveill Summ. 2015;64:125.

7. Palomba S, Homburg R, Santagni S, La Sala GB, Orvieto R. Risk of adverse pregnancy and perinatal outcomes after high technology infertility treatment: a comprehensive systematic review. Reprod Biol Endocrinol. 2016;14(1):76.

8. Messerlian C, Maclagan L, Basso O. Infertility and the risk of adverse pregnancy outcomes: a systematic review and meta-analysis. Hum Reprod. 2013;28:125-37.

9. Palomba S, Santagni S, Gibbins K, La Sala GB, Silver RM. Pregnancy complications in spontaneous and assisted conceptions of women with infertility and subfertility factors. A comprehensive review. Reprod Biomed Online. 2016;33(5):612-628.

10. Pinborg A, Wennerholm UB, Romundstad LB, Loft A, Aittomaki K, Soderstrom-Anttila V, et al. Why do singletons conceived after assisted reproduction technology have adverse perinatal outcome? Systematic review and meta-analysis. Hum Reprod Update. 2013;19:87-104.
11. Sunkara SK, LaMarca A, Polyzos NP, Seed PT, Khalaf $Y$. Live birth and perinatal outcomes following stimulated and unstimulated IVF: Analysis of over two decades of a nationwide data. Hum Reprod 2016;31:2261-7.

12. Kamath MS, Kirubakaran R, Mascarenhas M, Sunkara SK. Perinatal outcomes after stimulated versus natural cycle IVF: A systematic review and meta-analysis. Reprod Biomed Online 2018;36:94-101.

13. Pereira N, Reichman DE, Goldschlag DE, Lekovich JP, Rosenwaks Z. Impact of elevated peak serum estradiol levels during controlled ovarian hyperstimulation on the birthweight of term singletons from fresh IVF-ET cycles. J Assist Reprod Genet 2015;32:527-32.

14. Farhi J, Ben-Haroush A, Andrawus N, Pinkas $\mathrm{H}$, Sapir O, Fisch B, et al. High serum oestradiol concentrations in IVF cycles increase the risk of pregnancy complications related to abnormal placentation. Reprod Biomed. 2010;21:331-7.

15. Imudia AN, Awonuga AO, Doyle JO, Kaimal AJ, Wright DL, Toth TL, et al. Peak serum estradiol level during controlled ovarian hyperstimulation is associated with increased risk of small for gestational age and preeclampsia in singleton pregnancies after in vitro fertilization. Fertil Steril. 2012;97:1374-9.

16. Sunkara SK, La Marca A, Seed PT, Khalaf Y. Increased risk of preterm birth and low birthweight with very high number of oocytes following IVF: An analysis of 65868 singleton live birth outcomes. Hum Reprod 2015;30:1473-80.

17. Duan CC, Li C, He YC, Xu JJ, Shi CY, Hu HT, Su YF, Chen L, Tan YJ, Liu ZW, Sheng JZ, Fraser WD, Wu YT, Huang HF. Oocyte exposure to supraphysiological estradiol during ovarian stimulation increased the risk of adverse perinatal outcomes after frozen-thawed embryo transfer: a retrospective cohort study. J Dev Orig Health Dis. 2019;4:1-11.

18. Cai J, Liu L, Xu Y, Liu Z, Jiang X, Li P, et al. Estradiol level in ovarian stimulation cycles affects the birth weight of neonatal conceived through subsequent frozen-thawed cycles: A retrospective study. BJOG 2019;126:711-18.

19. Ombelet W, Peeraer K, De Sutter P, Gerris J, Bosmans E, Martens G, et al. Perinatal outcome of ICSI pregnancies compared with a matched group of natural conception pregnancies in 
flanders (Belgium): A cohort study. Reprod Biomed Online 2005;11:244-53.

20. Fedder J, Loft A, Parner ET, Rasmussen S, Pinborg A. Neonatal outcome and congenital malformations in children born after ICSI with testicular or epididymal sperm: A controlled national cohort study. Hum Reprod 2013;28:230-40.

21. Xiong X, Dickey RP, Buekens P, Shaffer JG, Pridjian G. Use of intracytoplasmic sperm injection and birth outcomes in women conceiving through in vitro fertilization. Paediatr Perinat Epidemiol 2017;31:108-15.

22. Maheshwari A, Hamilton M, Bhattacharya S. Should we be promoting embryo transfer at blastocyst stage? Reprod Biomed Online 2016;32:142-6.

23. Wang X, Du M, Guan Y, Wang B, Zhang J, Liu Z. Comparative neonatal outcomes in singleton births from blastocyst transfers or cleavage-stage embryo transfers: a systematic review and metaanalysis. Reprod Biol Endocrinol. 2017 May 4;15(1):36.

24. Alviggi C, Conforti A, Carbone IF, Borrelli R, de Placido G, Guerriero S. Influence of cryopreservation on perinatal outcome after blastocyst- vs cleavage-stage embryo transfer: systematic review and meta-analysis. Ultrasound Obstet Gynecol. 2018 Jan;51(1):54-63.

25. Marconi N, Raja EA, Bhattacharya S, Maheshwari A. Perinatal outcomes in singleton live births after fresh blastocyst-stage embryo transfer: a retrospective analysis of 67147 IVF/ICSI cycles. Hum Reprod. 2019 Sep 29;34(9):1716-1725.

26. Ginström Ernstad E, Spangmose AL, Opdahl S, Henningsen AA, Romundstad LB, Tiitinen A, Gissler M, Wennerholm UB, Pinborg A, Bergh C, Malchau SS. Perinatal and maternal outcome after vitrification of blastocysts: a Nordic study in singletons from the CoNARTaS group. Hum Reprod. 2019 Nov 1;34(11):2282-2289.

27. Mani S, Mainigi M. Embryo culture conditions and the epigenome. Semin Reprod Med 2018;36:211-220.

28. Rienzi L, Gracia C, Maggiulli R, LaBarbera AR, Kaser DJ, Ubaldi FM, et al. Oocyte, embryo and blastocyst cryopreservation in ART: systematic review and meta-analysis comparing slowfreezing versus vitrification to produce evidence for the development of global guidance. Hum Reprod Update. 2017;23:139-155.

29. Kuwayama M, Vajta G, Kato O, Leibo SP. Highly efficient vitrification method for cryopreservation of human oocytes. Reprod Biomed. 2005;11:3008.

30. Liu SY, Teng B, Fu J, Li X, Zheng Y, Sun XX. Obstetric and neonatal outcomes after transfer of vitrified early cleavage embryos. Hum Reprod. 2013;28:2093-10

31. Kaartinen N, Kananen K, Huhtala H, Keränen S, Tinkanen $\mathrm{H}$. The freezing method of cleavage stage embryos has no impact on the weight of the newborns. J Assist Reprod Genet. 2016;33:3939.

32. Gu F, Li S, Zheng L, Gu J, Li T, Du H, Gao C, Ding C, Quan S, Zhou C, Li P, Xu Y. Perinatal outcomes of singletons following vitrification versus slow-freezing of embryos: a multicenter cohort study using propensity score analysis. Hum Reprod. 2019;34(9):1788-1798.

33. Litzky JF, Boulet SL, Esfandiari N, Zhang Y, Kissin DM, Theiler RN, Marsit CJ. Effect of frozen/thawed embryo transfer on birthweight, macrosomia, and low birthweight rates in US singleton infants. Am J Obstet Gynecol 2018;218:433 e431-433 e410.

34. Maheshwari A, Pandey S, Amalraj Raja E, Shetty A, Hamilton M, Bhattacharya S. Is frozen embryo transfer better for mothers and babies? Can cumulative meta-analysis provide a definitive answer? Hum Reprod Update 2018;24:35-58.

35. Roque M, Haahr T, Geber S, Esteves SC, Humaidan P. Fresh versus elective frozen embryo transfer in IVF/ICSI cycles: a systematic review and meta-analysis of reproductive outcomes. Hum Reprod Update. 2019; 25:2-14.

36. Wei D, Liu JY, Sun Y, Shi Y, Zhang B, Liu JQ, et al. Frozen versus fresh single blastocyst transfer in ovulatory women: a multicentre, randomised controlled trial. Lancet. 2019 30;393:1310-1318.

37. Chen ZJ, Shi Y, Sun Y, Zhang B, Liang X, Cao Y, et al. Fresh versus Frozen Embryos for Infertility in the Polycystic Ovary Syndrome. N Engl J Med 2016;375:523-33.

38. Hiura $\mathrm{H}$, Hattori $\mathrm{H}$, Kobayashi $\mathrm{N}$, Okae $\mathrm{H}$, Chiba $H$, Miyauchi N, Kitamura A, Kikuchi $H$, Yoshida $H$, Arima T. Genome-wide microRNA expression profiling in placentae from frozen-thawed 
blastocyst transfer. Clin Epigenetics. 2017 Aug 3;9:79.

39. Zhang WY, von Versen-Höynck F, Kapphahn KI, Fleischmann RR, Zhao Q, Baker VL. Maternal and neonatal outcomes associated with trophectoderm biopsy. Fertil Steril. 2019 Aug;112(2):283-290.e2.

40. Sunkara SK, Antonisamy B, Selliah HY, Kamath MS. Pre-term birth and low birth weight following preimplantation genetic diagnosis: Analysis of 88 010 singleton live births following PGD and IVF cycles. Hum Reprod 2017;32:432-8.

41. Sauer MV, Paulson RJ, Lobo RA. Oocyte donation to women of advanced reproductive age: Pregnancy results and obstetrical outcomes in patients 45 years and older. Hum Reprod 1996;11:2540-3.

42. Zegers-Hochschild F, Schwarze JE, Crosby JA, Musri C, Urbina MT; Latin American Network of Assisted Reproduction (REDLARA). Assisted reproductive techniques in Latin America: the Latin American Registry 2016. Reprod Biomed Online. 2019;39:452-460.

43. De Geyter C, Calhaz-Jorge C, Kupka MS, Wyns C, Mocanu E, Motrenko T,et al. European IVFmonitoring Consortium (EIM) for the European Society of Human Reproduction and Embryology (ESHRE). ART in Europe, 2014: results generated from European registries by ESHRE: The European IVF-monitoring Consortium (EIM) for the European Society of Human Reproduction and Embryology (ESHRE). Hum Reprod. 2018; 33:1586-1601.

44. Malchau SS, Loft A, Larsen EC, Aaris Henningsen AK, Rasmussen S, Andersen AN, et al. Perinatal outcomes in 375 children born after oocyte donation: A Danish national cohort study. Fertil Steril 2013;99:1637-43.

45. Moreno-Sepulveda J, Checa MA. Risk of adverse perinatal outcomes after oocyte donation: a systematic review and meta-analysis. J Assist Reprod Genet. 2019 Oct;36(10):2017-2037.

46. Saito S, Nakabayashi Y, Nakashima A, Shima T, Yoshino O. A new era in reproductive medicine: consequences of third-party oocyte donation for maternal and fetal health. Semin Immunopathol. 2016 Nov;38(6):687-697.
47. Salha $O$, Sharma V, Dada $T$, Nugent $D$, Rutherford AJ, Tomlinson AJ, et al. The influence of donated gametes on the incidence of hypertensive disorders of pregnancy. Hum Reprod. 1999;14:2268-77.

48. Adams DH, Clark RA, Davies MJ, de Lacey S. A meta-analysis of sperm donation offspring health outcomes. J Dev Orig Health Dis. 2017 Feb;8(1):44-55.

49. Kawwass JF, Crawford S, Hipp HS, Boulet SL, Kissin DM, Jamieson DJ; National ART Surveillance System Group. Embryo donation: national trends and outcomes, 2000 through 2013. Am J Obstet Gynecol. 2016 Dec;215(6):747.e1-747.e5.

50. Brinsden PR. Gestational surrogacy. Hum Reprod Update. 2003;9:483-491.

51. Perkins KM, Boulet SL, Jamieson DJ, Kissin DM; National Assisted Reproductive Technology Surveillance System (NASS) Group. Trends and outcomes of gestational surrogacy in the United States. Fertil Steril 2016;106:435-4200.

52. Soderstrom-Anttila V, Wennerholm UB, Loft A, et al. Surrogacy: outcomes for surrogate mothers, children and the resulting families-a systematic review. Hum Reprod Update. 2016;22:260-276.

53. Sunkara SK, Antonisamy B, Selliah HY, Kamath MS. Perinatal outcomes after gestational surrogacy versus autologous IVF:Analysis of national data. Reprod Biomed Online 2017;35:708

54. Parkinson J, Tran C, Tan T, et al. Perinatal outcome after in-vitro fertilization-surrogacy. Hum Reprod. 1999;14:671-676.

55. Woo I, Hindoyan R, Landay M, et al. Perinatal outcomes after natural conception versus in vitro fertilization in gestational surrogates: a model to evaluate IVF treatment versus maternal effects. Fertil Steril. 2017;108:993-998.

56. Peters $H$, Schats $R$, Verhoeven $M$, et al. Gestational surrogacy: results of 10 years of experience in the Netherlands. Reprod Biomed Online. 2018;37:725-731. 